\title{
John Horton Conway (December 26, 1937-April 11, 2020)
}

丁

his special issue of the Mathematical Intelligencer is dedicated to the memory of John Horton Conway. Its articles describe Conway's contributions to many areas of mathematical research. Some also present new results inspired by his work that we believe he would like.

But this special issue is not just about research-level mathematics. Its articles also paint a portrait of a warm and generous man. Conway loved to discuss mathematics with whoever would listen, whether they were his mathematical peers or the many young students whom he inspired and with whom he gloried in playing mathematical games. You will see Conway both at play and at work in the pages that follow.

In many ways, it was natural for us to put together this special issue. Conway was a long-time friend of the Intelligencer. Over the years, he published, singly or with one or more coauthors, quite a number of papers in our journal. Among them:

- "Monsters and Moonshine." 4:2 (1980), 165-171.

- (With H. Dietrich and E. A. O’Brien) "Counting Groups: Gnus, Moas, and Other Exotica." 30:2 (2008), 6-18.

- (With A. Ryba) "The Pascal Mysticum Demystified." 34:3 (2012), 4-8.

- (With A. Ryba) "Extending the Pascal Mysticum." 35:2 (2013), 44-51.

- (With J. Shipman) "Extreme Proofs I: The Irrationality of $\sqrt{2} . " 35: 3$ (2013), 2-7.

- "A Characterization of the Equilateral Triangles and Some Consequences." 36:2 (2014), 1-2.

- "On Morley's Trisector Theorem." 36:3 (2014), 3.

- (With B. Heuer) "All Solutions to the Immobilizer Problem." 36:4 (2014), 78-86.

- (With C. Bae, L. Kohlhase, and S. Park) "Prague Clocks." 38:1 (2016), 37-39.

- (With A. Ryba) "The Extra Fibonacci Series and the Empire State Building." 38:1 (2016), 41-48.

- "Chemical $\pi$." 38:4 (2016), 7-10.

- (With K. Boklan) "Expect at Most One Billionth of a New Fermat Prime!" 39:1 (2017), 3-5.

- (With O. G. Cassani) "Neumbering." 40:1 (2018), 91-92.

We hope you enjoy this glimpse into the extraordinary life and work of John Horton Conway!

Karen Hunger Parshall Marjorie Wikler Senechal Sergei Tabachnikov 